\title{
Emprego Concomitante da Localização Radioguiada da Lesão e do Estudo do Linfonodo Sentinela para o Carcinoma Invasor de Mama Não Palpável
}

\author{
Nonpalpable Infiltrative Breast Cancer: Concomitant Use of Radioguided Occult \\ Lesion Localization and Sentinel Lymph Node Biopsy
}

José Roberto Morales Piato, Alfredo Carlos Simões Dornelas de Barros, Antonio Carlos Toshihiro Nisida, Carlos Alberto Buchpigel, Nestor de Barros, José Aristodemo Pinotti

\begin{abstract}
RESUMO
Objetivo: analisar o emprego concomitante da localização radioguiada de lesão não palpável e do estudo do linfonodo sentinela (LS) em mulheres com câncer de mama.

Método: estudo prospectivo de 45 pacientes com carcinoma não palpável de mama. $O$ radiofármaco utilizado foi o dextram marcado pelo ${ }^{99 m} T c$. Realizou-se injeção no sítio peritumoral, sob orientação ultra-sonográfica ou estereotáxica, com auxílio de agulha de raquianestesia de gauge 18. A linfocintilografia, para visualização pré-operatória do local da lesão e do LS, foi feita em gama câmera convencional, com abdução de $90^{\circ}$ do membro superior ipsilateral. A exérese da lesão primária e a do $L S$ foram realizadas com auxílio de sonda de detecção de radiação gama.

Resultados: quanto à remoção do tumor primário, obteve-se taxa de sucesso em todos os casos. Houve necessidade de ampliação de margem cirúrgica em cinco pacientes. Em relação ao LS houve falha em quatro casos, com indice de localização de 93\%. Não foram observadas complicações.

Conclusão: os resultados obtidos parecem demonstrar que a utilização simultânea dos dois procedimentos constitui estratégia eficaz para o tratamento do câncer inicial de mama em uma única sessão
\end{abstract}

PALAVRAS-CHAVE: Mama: câncer. Carcinoma não palpável de mama. Linfonodo sentinela.

Introdução

O uso alargado da mamografia no rastreamento do câncer de mama tem possibilitado a detecção de número crescente de lesões não palpáveis. Entre $0,5 \%$ e $2 \%$ das mulheres assintomáticas submetidas a controle mamográfico têm biópsia indicada devido ao achado de lesão oculta suspeita ${ }^{1}$. Estima-se que num futuro

Hospital das Clínicas da Faculdade de Medicina da USP Clinica Ginecológica, Departamento de Medicina Nuclear e Departamento de Radiologia

Correspondência:

José Roberto M. Piato

Rua Conselheiro Brotero, 1539, conj. 43

01232-011 - São Paulo - SP

Fone/Fax: (11) 3825-4455

e-mail: jrpiato@uol.com.br próximo, cerca de $50 \%$ dos cânceres mamários serão diagnosticados na fase pré-clínica ${ }^{2}$.

Durante muitos anos, a metodologia utilizada para a localização da lesão, com vista à prática de biópsia, consistia na inserção de fio metálico, guiada por exame ultra-sonográfico ou por estereotaxia ${ }^{3}$. Em 1998 foi descrito novo método de localização de lesões mamárias não palpáveis, o qual recebeu a denominação ROLL (radioguided occult lesion localization) ${ }^{4}$. A técnica proposta consiste na injeção intratumoral, orientada por ultrasonografia ou por mamografia, de $0,2 \mathrm{~mL}$ de albumina coloidal marcada com ${ }^{99 \mathrm{~m}} \mathrm{Tc}$ e na utilização de detector de radiação gama (sonda) para localização intra-operatória da lesão.

Até recentemente, o estudo do linfonodo sentinela (LS) em mulheres com lesão mamária não palpável era realizado em segundo tempo, após o 
diagnóstico definitivo do carcinoma. Assim, na eventualidade de se ter utilizado o ROLL ou outra técnica para excisão de lesões não palpáveis, torna-se necessária nova injeção de radiofármaco, próximo do local da biópsia, para localização do $\mathrm{LS}^{5,6}$.

Pela análise da literatura verifica-se que nos últimos anos alguns autores têm utilizado a associação do método ROLL com o estudo do $\mathrm{LS}^{7-9}$. Os trabalhos realizados por estes autores evidenciam que esta estratégia apresenta bons resultados, com indices de localização da lesão primária de 100\% e de localização do LS entre 97,3\% e 100\%; demonstrando, desta forma, pouca divergência. $\mathrm{O}$ presente trabalho tem como objetivo analisar a viabilidade de emprego desta metodologia em estudo prospectivo.

\section{Pacientes e Métodos}

Na Clínica Ginecológica da Faculdade de Medicina da USP iniciamos a utilização do método ROLL em 1998. Desde esta época estamos realizando sua prática concomitantemente com o estudo do linfonodo sentinela. O presente estudo prospectivo incluiu 45 pacientes afetadas por câncer inicial de mama, assistidas na Clínica Ginecológica da Faculdade de Medicina da USP, no período entre novembro de 1998 e fevereiro de 2003. Os métodos utilizados para o diagnóstico foram biópsia com agulha grossa ou exame intraoperatório por congelação. O diâmetro da lesão primária não ultrapassou $1,5 \mathrm{~cm}$, sendo que a mensuração foi realizada no momento da cirurgia pelo patologista, com auxílio de régua comum.

O projeto do trabalho foi aprovado pelo Comitê de Ética em Pesquisa do Hospital das Clínicas da Faculdade de Medicina da USP.

O material utilizado para a localização radioguiada das lesões não palpáveis da mama (ROLL) e para o estudo simultâneo do LS consistiu em mamógrafo ou aparelho de ultra-sonografia, agulha de raquianestesia de gauge 18, material radioativo, gama-câmara convencional e aparelho portátil de detecção de radiação gama.

A substância radioativa empregada para o método ROLL e para o estudo concomitante do LS foi o dextram 70 , marcado pelo ${ }^{99 \mathrm{~m}} \mathrm{Tc}$, com atividade de 0,4 mCi. A gama-câmara consistiu em câmara de cintilação equipada com colimador de baixa energia, calibrado para ajuste do fotopico em $140 \mathrm{keV}$, com janela de $20 \%$. Para detecção da emissão radioativa utilizamos sonda de captação de radiação gama.

Com vista à injeção do radiofármaco, a localização da lesão oculta da mama foi feita por meio de ultra-sonografia ou de mamografia. Nos casos em que utilizamos ultra-sonografia, a injeção do material radioativo foi orientada pela imagem em tempo real; nas demais pacientes, em que não se observou expressão ultra-sonográfica da lesão, empregamos método estereotáxico. Nas mesmas, o primeiro passo consistiu na realização de mamografia de base, para estabelecer a topografia da lesão. Uma vez feita a localização, a paciente foi mantida imobilizada na mesa de exame, para a prática da estereotaxia, que foi feita pela técnica biplanar, com auxilio de compressor fenestrado.

Após anti-sepsia e anestesia local intradérmica da pele correspondente à área de projeção da lesão, procedemos a introdução da agulha de raquianestesia, procurando posicionar a extremidade distal da mesma na região justatumoral. Para verificar se este posicionamento foi realizado corretamente, realizamos controle radiológico nas projeções perfil e crânio-caudal.

A etapa seguinte consistiu na injeção de 0,2 $\mathrm{mL}$ de dextram marcado pelo ${ }^{99 \mathrm{~m} T c}$. Para evitar contaminação retrógrada pelo radiofármaco, mantivemos a mama ainda comprimida durante a prática da injeção, retirando a agulha em seguida.

Após a injeção do material radioativo foi realizada linfocintilografia na gama-câmara convencional. Para a obtenção das imagens cintilográficas, as pacientes foram posicionadas com o membro superior ipsilateral em abdução de 90 graus, mesma posição em que se realizou posteriormente a cirurgia. As imagens estáticas foram adquiridas com tempo que variou entre 180 e 300 segundos por projeção, nas incidências anterior, obliqua-anterior e perfil (Figura 1). Após aquisição das imagens utilizou-se fonte radioativa pontual, a fim de identificar a projeção cutânea do linfonodo sentinela, com dermografia da área.

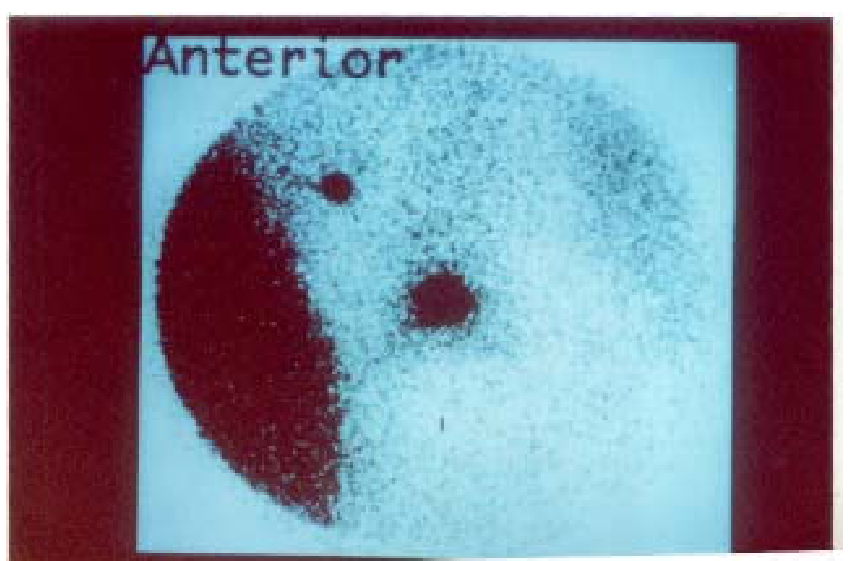

Figura 1 - Cintilografia em projeção oblíqua-anterior, mostrando imagem do sítio de injeção (maior) e da área de captação axilar (menor). 
No pré-operatório imediato utilizamos a sonda para verificar se as áreas de marcação na pele correspondiam àquelas de maior contagem em sinal acústico, tanto para a lesão primária (ROLL) quanto para o linfonodo sentinela.

Para a remoção da lesão primária demos preferência à incisão periareolar, visando melhores resultados cosméticos. A exérese foi realizada sob controle constante pela sonda, tendo em vista a tridimensionalidade da lesão. Nos casos em que houve persistência de atividade radioativa residual em um ou mais locais do leito cirúrgico, ampliou-se a ressecção. Com a finalidade de orientar a avaliação das margens do espécime cirúrgico, pelo patologista, procedeu-se a sua marcação com fios de algodão, em diferentes tamanhos. A confirmação da completa retirada da lesão foi realizada por meio de radiografia do espécime cirúrgico em mamógrafo. $\mathrm{O}$ exame intra-operatório das margens foi realizado por cortes histológicos e/ou imprints citológicos.

A biópsia do linfonodo sentinela foi realizada na seqüência. Sempre que possivel, utilizamos a mesma incisão empregada no ROLL, localizando o linfonodo captante com auxílio da sonda. Havendo dificuldades técnicas, empregamos incisão separada. Para facilitar a localização do LS, expusemos sistematicamente o cavo axilar, através da abertura da fáscia clavipeitoral, ao longo das bordas dos músculos grande e pequeno peitorais.

Nos primeiros 21 casos realizamos dissecção axilar completa, independentemente do estado do LS. Nos demais, a dissecção axilar foi realizada somente em três pacientes em que o LS apresentava-se comprometido por metástases.

Não utilizamos drenos, seja na cirurgia mamária ou na exérese do linfonodo sentinela. Foi realizada antibioticoterapia profilática com cefalosporina de primeira geração, na dose de $2 \mathrm{~g}$.

\section{Resultados}

Em relação à localização radioguiada da lesão (ROLL), obtivemos sucesso em todas as pacientes. Em cinco pacientes, nas quais o exame pelo patologista revelou comprometimento de margens cirúrgicas, procedeu-se a ampliação das mesmas.

Quanto ao estudo do linfonodo sentinela, ocorreu falha do método em quatro casos, com indice de acurácia de $93 \%$. As quatro pacientes em que não foi possivel localizar o LS tinham idade superior a 65 anos.

O tempo médio de duração da exérese do tumor primário foi de 25 minutos e o da biópsia do linfonodo sentinela de 30 minutos.
No que se relaciona ao ROLL, não tivemos dificuldades técnicas. Quanto à exérese do LS, surgiram pequenas dificuldades nos casos em que o mesmo encontrava-se em localização profunda no cavo axilar.

Em nenhuma das pacientes observaram-se complicações intra ou pós-operatórias.

\section{Discussão}

O emprego de fio metálico para localização de lesões não palpáveis da mama apresenta alguns inconvenientes relevantes, tais como riscos de posicionamento errôneo do fio e de mudança de sua posição correta, especialmente em mamas gordurosas, e excessiva remoção de tecido mamário.

As vantagens do método ROLL em relação ao fio metálico, quanto à eficácia, têm sido evidenciadas em alguns trabalhos. No estudo pioneiro sobre ROLL, que envolveu 225 pacientes, os idealizadores do método verificaram que a radiografia do espécime cirúrgico proporcionou sucesso na ressecção da lesão em $99 \%$ das vezes ${ }^{4}$. Em outra avaliação, que envolveu 816 casos de lesão mamária não palpável, foi observada eficácia do ROLL em 94,6\% dos $\operatorname{casos}^{10}$. Em nosso meio, estudo de 38 mulheres com lesões mamárias suspeitas não palpáveis (BIRADS 4 e 5), este método possibilitou a remoção da lesão em todas as vezes ${ }^{9}$.

Cabe ressaltar que o método ROLL apresenta outras vantagens em relação ao fio metálico. Uma das mesmas é possibilitar a preservação de maior quantidade de tecido sadio entre a pele da mama e a lesão; graças a isto, os resultados estéticos são mais favoráveis ${ }^{11,12}$. Finalmente, tem-se verificado que, com o emprego desta técnica, o tumor comumente apresenta-se eqüidistante de todas as margens do espécime cirúrgico, o que facilita seu estudo intra-operatório pelo patologista.

Quanto à utilização simultânea do ROLL e do estudo do linfonodo sentinela existem dados na literatura que, igualmente, mostram elevadas taxas de eficácia. Em estudo de 44 pacientes com câncer infiltrativo de mama obteve-se sucesso quanto à localização (ressecção) das lesões em todos os casos com o emprego da localização radioguiada ${ }^{13}$. No que se refere à localização concomitante do LS, o índice de eficácia foi de $98 \%{ }^{8}$. Em outro trabalho, no qual foram incluídas 73 pacientes, as referidas taxas foram 100\% e 97,3\%, respectivamente ${ }^{7}$. Em 11 pacientes de nosso meio, com diagnóstico de carcinoma invasor, nas quais empregou-se simultaneamente ROLL e estudo do LS, foi observado sucesso da metodologia em todos os $\operatorname{casos}^{9}$. 
Com vista à obtenção de bons resultados no emprego simultâneo do método ROLL e do estudo do LS, deve-se utilizar material radioativo e instrumental adequados e devem ser obedecidos com rigor certos detalhes da técnica. O ${ }^{99 \mathrm{~m}} \mathrm{Tc}$ é considerado o radiofármaco de eleição, uma vez que apresenta características ideais para a localização da lesão e do linfonodo sentinela, isto é, energia de imagem apropriada, meia-vida física de apenas seis horas, baixo custo e ampla disponibilidade ${ }^{14}$.

Em relação ao estudo do LS, é importante que se utilize material radioativo cujas partículas possuam tamanho adequado. Em trabalho, no qual se empregou albumina humana, com partículas medindo entre 200 e $1.000 \mathrm{~nm}$, verificou-se relação inversamente proporcional entre o tamanho da partícula e o número de linfonodos detectados pela linfocintilografia ${ }^{15}$. Quanto ao dextram, desconhece-se o tamanho da partícula. Supõe-se que o mesmo esteja dentro do referido intervalo, uma vez que o número de linfonodos sentinelas marcados é semelhante ${ }^{16}$.

No que se refere aos métodos de imagem destinados à orientação do local para injeção do material radioativo, a ultra-sonografia deve ser utilizada de preferência, quando a lesão pode ser evidenciada por este método ${ }^{16}$.

Durante a injeção do material radioativo, que deve ser feita na periferia do tumor, é necessário que se mantenha compressão da mama, para reduzir o risco decorrente de mudança de posição da extremidade da agulha. Com este cuidado evita-se contaminação retrógrada pelo radiofármaco, no ato de remoção da agulha.

Para que a captação das radiações gama seja convertida em leitura digital e sinal sonoro de intensidade e freqüência diretamente proporcionais ao nivel de radiação emitida, é necessária a utilização de sonda de alta qualidade. Esta deve apresentar boa resolução espacial, para evitar interferências decorrentes da proximidade entre o sítio de injeção do radiofármaco e o linfonodo sentinela captante ${ }^{17}$. Tal fato tem maior relevância nos casos em que o tumor se localiza no quadrante superior lateral.

O exame radiográfico do espécime cirúrgico deve ser realizado sistematicamente, para verificar se houve remoção completa da lesão. Esta medida é especialmente útil nos casos em que a lesão primária é constituída por microcalcificações.

Os resultados por nós obtidos com metodologia que associa localização radioguiada de carcinomas não palpáveis e estudo do linfonodo sentinela, obedecendo os princípios acima assinalados, assemelham-se àqueles referidos por outros autores. Assim, obteve-se a localização da lesão em todas as pacientes, enquanto a taxa de localização do linfonodo sentinela foi de $93 \%$. Nas quatro pacientes em que ocorreu falha em localizar o LS, possivelmente o fato deveu-se a alterações da circulação linfática das mesmas, em decorrência da idade avançada.

Nossa conclusão é de que o emprego concomitante do método ROLL e do estudo do linfonodo sentinela, em mulheres afetadas por câncer invasor não palpável de mama, proporcionou índices de localização da lesão primária e do LS semelhantes àqueles usualmente obtidos com o uso desses procedimentos em tempos separados. Assinalamos ainda que esta nova estratégia, por ser realizada em tempo único, pode ser útil no sentido de reduzir a ansiedade da paciente. Por fim, torna-se evidente que os custos tornam-se menores, uma vez que se lança mão dos recursos de medicina nuclear uma única vez.

\section{ABSTRACT}

Purpose: to assess the simultaneous approach of radioguided occult lesion localization and sentinel lymph node biopsy in women with breast cancer.

Method: forty-five patients with nonpalpable breast cancer were enrolled in a prospective study. The employed radiocolloid was ${ }^{99 m} T c$-labelled dextran. The injection was performed peritumorally under sonographic or stereotactic guidance using a 18 gauge needle. Lymphoscintigraph images for the visualization of both the site of injection and sentinel lymph node were obtained in a conventional gammacamera, with the ipsilateral arm positioned in a $90^{\circ}$ angle. Excision biopsy of the tumor and sentinel lymph node were performed with a gamma-detecting probe.

Results: the procedure was always successful in permitting the localization of occult breast lesions. It was necessary to enlarge surgical margins in five cases. Concerning the sentinel lymph node we achieved localization in 93\% of the cases. No complications were observed.

Conclusion: the results seems to demonstrate that a combined radioguided occult lesion localization and sentinel lymph node biopsy using the same radiopharmaceutical represents a useful and practicable strategy in the management of early breast cancer.

KEYWORDS: Breast: neoplasms. Nonpalpable breast cancer. Sentinel lymph node. 


\section{Referências}

1. Hall FM, Storella JM, Silverstone DZ, Wyshak G. Nonpalpable breast lesions: recommendations for biopsy based on suspicion of carcinoma at mammography. Radiology 1988; 167:353-8.

2. Tubiana M, Holland R, Kopans DB, et al. Management of non-palpable and small lesions found in mass breast screening. Commission of European Communities "Europe Against Cancer Programme". European School of Oncology Advisory Report. Eur J Cancer 1994; 30A:538-47.

3. Kopans DB, DeLuca S. A modified needle-hookwire technique to simplify preoperative localization of occult breast lesions. Radiology 1980; 134:781.

4. Zurrida S, Galimberti V, Monti S, Luini A. Radioguided localization of occult breast lesions. Breast 1998; 7:11-3.

5. Veronesi U, Paganelli G, Galimberti V, et al. Sentinelnode biopsy to avoid axillary dissection in breast cancer with clinically negative lymph-nodes. Lancet 1997; 349:1864-7.

6. Borgstein PJ, Pijpers R, Comans EF, van Diest PJ, Boom RP, Meijer S. Sentinel lymph node biopsy in breast cancer: guidelines and pitfalls of lymphoscintigraphy and gamma probe detection. J Am Coll Surg 1998; 186:275-83.

7. Feggi L, Basaglia E, Corcione S, et al. An original approach in the diagnosis of early breast cancer: use of the same radiopharmaceutical for both nonpalpable lesions and sentinel node localization. Eur J Nucl Med 2001; 28:1589-96.
8. Gray RJ, Giuliano R, Dauway EL, Cox CE, Reintgen DS. Radioguidance for nonpalpable primary lesions and sentinel lymph node(s). Am J Surg 2001; 182:404-6.

9. Barros A, Cardoso MA, Sheng PY, Costa PA, Pelizon C. Radioguided localization of non-palpable breast lesions and simultaneous sentinel lymph node mapping. Eur J Nucl Med Mol Imaging 2002; 29:1561-5.

10.De Cicco C, Pizzamiglio M, Trifirò G, et al. Radioguided occult lesion localization (ROLL) and surgical biopsy in breast cancer. Technical aspects. Q J Nucl Med 2002; 46:145-51.

11.Burelli P, Marinelli P, Borsato N, Bedin N. Metodica abbinata roll-linfonodo sentinela: nuove strategie della chirurgia radioguidata. Chir Ital 2002; 54:20912 .

12.Luini A, Zurrida S, Paganelli G, et al. Comparison of radioguided excision with wire localization of occult breast lesions. Br J Surg 1999; 86:522-5.

13.Paganelli G, Veronesi U. Innovation in early breast cancer surgery: radio-guided occult lesion localization and sentinel node biopsy. Nucl Med Commun 2002; 23:625-7.

14.Paganelli G, de Cicco C, Cremonesi M, et al. Optimized sentinel node scintigraphy in breast cancer. Q J Nucl Med 1998; 42:49-53.

15. Offodile R, Hoh C, Barsky SH, et al. Minimally invasive breast carcinoma staging using lymphatic mapping with radiolabeled dextran. Cancer 1998; 82:1704-8.

16.Keshtgar MR, Ell PJ. Sentinel lymph node detection and imaging. Eur J Nucl Med 1999; 26:57-67.

17.Britten AJ. A method to evaluate intra-operative gamma probes for sentinel lymph node localization. Eur J Nucl Med 1999; 26:76-83. 